\title{
Academic stress and personality interact to increase the neural response to high-calorie food cues
}

\author{
Selin Neseliler ${ }^{a}$, Beth Tannenbaum ${ }^{a}$, Maria Zacchia ${ }^{a}$, Kevin Larcher ${ }^{a}$, Kirsty Coulter ${ }^{a}$, \\ Marie Lamarche ${ }^{\mathrm{b}}$, Errol B. Marliss ${ }^{\mathrm{b}}$, Jens Pruessner ${ }^{\mathrm{a}, \mathrm{c}}$, Alain Dagher ${ }^{\mathrm{a}}$ * \\ ${ }^{a}$ Montreal Neurological Institute, McGill University, Montréal, Canada

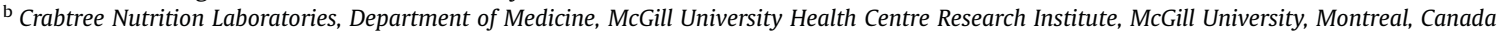 \\ ${ }^{c}$ Douglas Mental Health University Institute, McGill University, Montreal, Canada
}

Keywords:

Psychosocial stress

Food cue reactivity

Obesity

Neuroimaging

Personality

\begin{abstract}
A B S T R A C T
Psychosocial stress is associated with an increased intake of palatable foods and weight gain in stressreactive individuals. Personality traits have been shown to predict stress-reactivity. However, it is not known if personality traits influence brain activity in regions implicated in appetite control during psychosocial stress. The current study assessed whether Gray's Behavioural Inhibition System (BIS) scale, a measure of stress-reactivity, was related to the activity of brain regions implicated in appetite control during a stressful period. Twenty-two undergraduate students participated in a functional magnetic resonance imaging (fMRI) experiment once during a non-exam period and once during final exams in a counter-balanced order. In the scanner, they viewed food and scenery pictures. In the exam compared with the non-exam condition, BIS scores related to increased perceived stress and correlated with increased blood-oxygen-level dependent (BOLD) response to high-calorie food images in regions implicated in food reward and subjective value, such as the ventromedial prefrontal cortex, (VmPFC) and the amygdala. BIS scores negatively related to the functional connectivity between the vmPFC and the dorsolateral prefrontal cortex. The results demonstrate that the BIS trait influences stress reactivity. This is observed both as an increased activity in brain regions implicated in computing the value of food cues and decreased connectivity of these regions to prefrontal regions implicated in self-control. This suggests that the effects of real life stress on appetitive brain function and self-control is modulated by a personality trait. This may help to explain why stressful periods can lead to overeating in vulnerable individuals.
\end{abstract}

\section{Introduction}

Psychosocial stress has been linked to unhealthy eating and weight gain, but only for some individuals. Differences in stress vulnerability can explain this heterogeneity (Adam \& Epel, 2007; Dallman, 2010; Groesz et al., 2012; Newman, O'Connor, \& Conner, 2007; Tomiyama, Dallman, \& Epel, 2011). The purpose of this study was to study the neural correlates of individual differences in stress reactivity on the brain circuits implicated in food intake.

Individual reactivity to psychological stress can be assessed via perceived stress questionnaires or by measuring levels of plasma or

* Corresponding author. Montreal Neurological Institute, 3801 University St., Montréal, QC H3A 2B4, Canada.

E-mail address: alain.dagher@mcgill.ca (A. Dagher). salivary cortisol, a reflection of hypothalamo-pituitaryadrenocortical (HPA) axis activation (McEwen et al., 2015). Greater stress reactivity by either measure is associated with increased high-calorie food consumption outside of the laboratory (Groesz et al., 2012; Newman et al., 2007) and greater body mass index (BMI) (Tomiyama et al., 2011). The relationship between stress and weight gain in longitudinal studies has been more variable suggesting that there might be additional factors influencing this relationship (Wardle, Chida, Gibson, Whitaker, \& Steptoe, 2011).

Personality traits have been proposed as a reflection of the vulnerability to stress. Stress reactivity has been related to scores on the Behavioural Inhibition and Activation System (BIS/BAS) scales of Carver and White (Carver \& White, 1994a). The BIS measure of the BIS/BAS scale, as detailed in Gray's Reinforcement Sensitivity Theory, assesses an individual's sensitivity to 
punishments and aversive events (Gray, 1987), and correlates with the similar measure of Neuroticism from the Five Factor Model of personality (Keiser \& Ross, 2011). For example, in one longitudinal study, BIS scores moderated the relationship between violence exposure and post-traumatic stress disorder symptoms (Gudiño, 2013). High scores in BIS are also related to increased perceived stress levels at work (van der Linden, Taris, Beckers, \& Kindt, 2007) and eating disturbances in college students (Chang, Kahle, Yu, \& Hirsch, 2014).

Heightened reactivity to stressors can increase the probability of self-control failure during food choice, especially for caloricallydense food items (Epel, Lapidus, McEwen, \& Brownell, 2001; Maier, Makwana, \& Hare, 2015; Tomiyama et al., 2011). Such impulsive behavior may be mediated by increased food-reward sensitivity and decreased executive function, features that have been reported consistently in the obesity literature (Vainik, Dagher, Dubé, \& Fellows, 2013). In fMRI experiments, heighted reward sensitivity may be reflected as increased BOLD within the appetitive brain network while viewing high-calorie food pictures. This set of limbic and striatal brain regions is involved in coding the incentive salience of foods and engaging appropriate motor responses. It includes the hippocampus, the amygdala, the insula, the ventral and dorsal striatum, and the orbitofrontal cortex (OFC) and adjacent vmPFC (Dagher, 2012). For example, acute stress led to increased BOLD in the right amygdala upon ingestion of a highcalorie milkshake (Rudenga, Sinha, \& Small, 2012), and increased connectivity of the vmPFC to the amygdala and ventral striatum when choosing a high-calorie over a healthy food item (Maier et al., 2015). These effects correlated with individuals' stress reactivity (i.e. changes in cortisol levels). On the other hand, decreased executive function, possibly reflecting poor self-control, has been associated to reduced activity of the dorsolateral prefrontal cortex (dIPFC) and the dorsal anterior cingulate cortex (dACC) (VerdejoGarcia, 2014). Perceived stress during an acute laboratory stressor led to reduced functional connectivity between dIPFC and vmPFC during a food choice task that demanded self-control (Maier et al., 2015). This was thought to reflect a stress-induced impairment in self-regulation during food choice.

Effects of chronic stress on food intake may also work through a similar mechanism. For example, one cross-sectional study has shown that chronic stress load predicted greater BOLD signal in the appetitive regions and reduced activity in lateral PFC while viewing appetizing food pictures (Tryon, Carter, Decant, \& Laugero, 2013). However, cross-sectional studies cannot directly address the cause and effect relationship between stress and brain reactivity, and whether personality traits might modulate this relationship.

We studied undergraduate university students on two occasions: during the final exam period and during the school year, to measure within-subject effects of subacute stress on the brain mechanisms of appetite control. We hypothesized that the BIS score would predict individual differences in stress reactivity and that this would be reflected in the fMRI response to high-calorie food stimuli in appetitive and executive brain regions.

\section{Materials and methods}

\subsection{Participants}

22 right-handed undergraduate students [13 females, $\mathrm{BMI}=22.64(\mathrm{SD}= \pm 1.9)$; age $=20.5(\mathrm{SD}= \pm 2.9)]$ participated in the experiments. Participants were recruited via advertisements posted on campus and on online school classifieds at the beginning of each semester. Exclusion criteria included moderate or severe depression (score $>13$ measured by the 13-item Beck Depression Inventory (Beck A.T, 1972)), BMI>27, current cigarette smoking, history of substance abuse and current use of a central nervous system active medication, any history of neurological, psychiatric or eating disorders, being currently on a weight-reducing diet, and food restrictions due to allergies, intolerance or vegetarianism. The study was approved by the Montreal Neurological Institute Research Ethics Board. Each subject signed an approved informed consent form and received compensation for participation.

\subsection{Experimental Design}

Participants underwent fMRI on two occasions: once during the final exam period, and once during a non-exam period of the academic year. For the academic stress session, participants were required to have a minimum of three written final exams, two of which had to be scheduled within 5 days following the scanning day. The order of the scanning sessions was counterbalanced across participants and all of the scans started in the afternoon between 2:00 p.m. and 5:00 p.m. to account for diurnal changes in hormone levels and appetite. Scanning sessions were scheduled one month apart at the same time of day for each participant. Female participants were scanned during their luteal phase. On the scan days, participants were asked to refrain from exercise and caffeine, and were instructed not to eat anything for four hours prior to the experimental session. At arrival in the lab, they were given the same standard lunch (turkey and cheese sandwich, yogurt and fruit, $535 \mathrm{kcal}$ ). Participants were instructed to finish their meal, but three participants did not completely consume it in one session. Participants underwent fMRI two hours after eating. Blood samples were obtained immediately prior to and immediately following the fMRI scan to assess plasma levels of ghrelin (Fig. 1).

\subsection{Psychological measures}

Participants filled out the Three Factor Eating Questionnaire (TFEQ), a measure of eating styles (Stunkard \& Messick, 1985), as well as the BIS/BAS Scale (Carver \& White, 1994b). The BIS scores reported here are the mean of the two sessions. Scores showed good test-retest reliability across sessions $(r=0.85)$.

The self-report Perceived Stress Scale (PSS) was administered at the start of each session (Cohen, Kamarck, \& Mermelstein, 1983). It is a measure of an individual's perception of chronic stress during the last month. In addition, acute stress and hunger levels were assessed using Visual Analog Scales (VAS) throughout the scanning session. On the VAS, we asked the participants "On a scale from 0 to 10 how hungry/stressed do you feel now?"

\subsection{Hormone measurements}

Saliva samples were collected to measure diurnal cortisol levels for 4 days per session (exam and non-exam). For each session, subjects were instructed to collect their own saliva samples using salivettes ${ }^{\mathbb{B}}$ (Sarstedt Inc., Rommelsdorf, Germany) on two weekdays and one weekend day in the week preceding the scan, as well on the day of the scan (Fig. 1). Sampling was conducted five times on each day: upon awakening, 30 min after awakening, at lunch, at dinner, and at bedtime (Supplemental Table 4). They were asked to refrigerate the samples immediately after collection, in containers provided. Levels of salivary cortisol were measured using a timeresolved fluorescence immunoassay (Kirschbaum \& Hellhammer, 1994) and log-transformed. We measured cortisol awakening response (CAR) which is characterized by a sharp increase within the first 30 min after awakening. CAR provides a marker of current allostatic stress load (J. C. Pruessner et al., 1997). It can be calculated using the area under the curve (AUC) method that yields measures with respect to ground (AUCg) and baseline (AUCi) values (Jens $C$ 


\section{EXPERIMENTAL DESIGN}

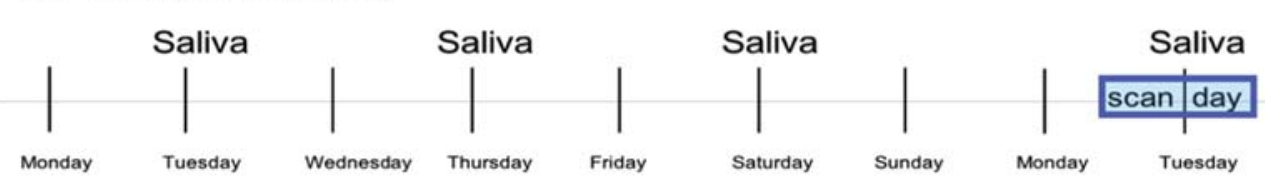

\section{$\underline{\text { SCAN DAY }}$}

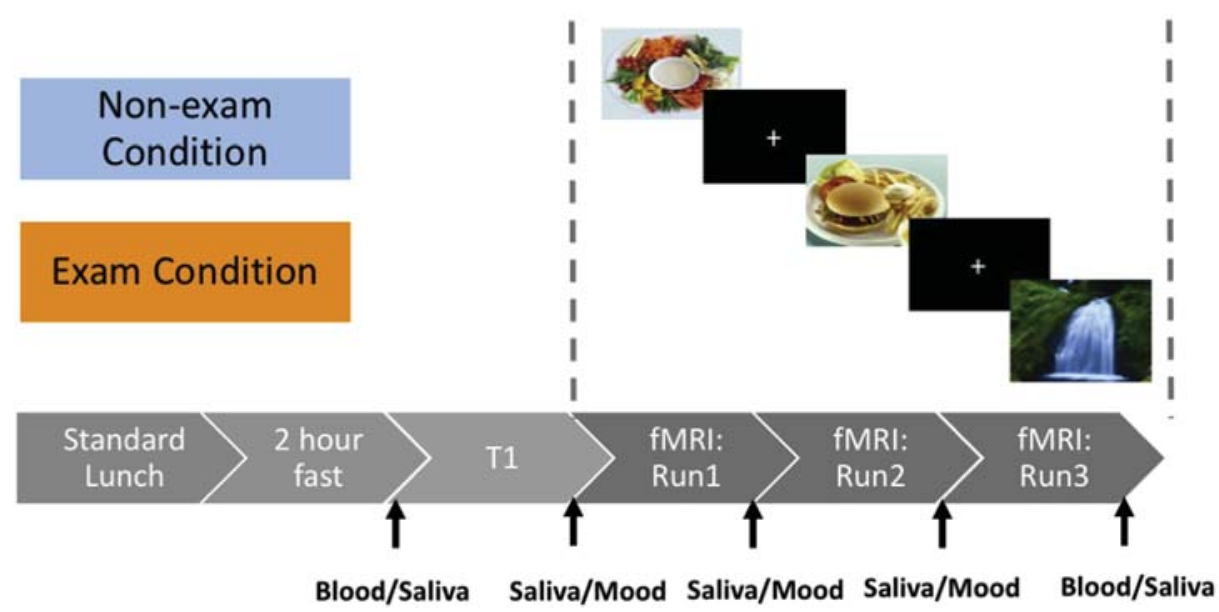

Fig. 1. Experimental design.

Participants $(\mathrm{N}=22)$ were tested twice, once during the non-exam period of the school year and once during final exams. Participants were asked to collect their saliva samples using salivettes ${ }^{\circledR}$ for four days leading up to each scan session (exam and non-exam). The order of the exam and non-exam conditions was counter-balanced. On each scan day, participants were given a standard lunch two hours prior to fMRI imaging. During fMRI they viewed pictures of low or high-calorie foods and scenery.

Pruessner, Kirschbaum, Meinlschmid, \& Hellhammer, 2003). AUCg has been associated with total hormone secretion while AUCi has been utilized to assess the rate of change of cortisol over time. In addition, we collected saliva five times during each scanning session: before and after the scan and before each functional run during the scan (Fig. 1). Different from the at-home samples, the laboratory measurements of cortisol were collected by the investigators at the same time of the day across sessions for each participant. We calculated the AUCi levels, taking the after the scan (5th) time point as the baseline, to determine the rate of change in cortisol rhythm between exam and non-exam scan sessions.

Both total and acylated ghrelin levels were measured from the blood samples collected prior to and after the scan and stored at $-80{ }^{\circ} \mathrm{C}$ until analysis by radioimmunoassay (Millipore, Billerica, MA,USA) (Pemberton, Wimalasena, Yandle, Soule, \& Richards, 2003). Only 15 ghrelin samples were included in the analysis due to improper storage conditions. The statistical analysis of hormonal and psychological measures was conducted using MATLAB (Version R2015a, MathWorks, Inc., Natick, MA, USA). These longitudinal analyses were run using linear mixed modelling (Matlab function fitlme) with subject as a random effect.

\subsection{FMRI acquisition}

Neuroimaging was carried out with a Siemens Magnetom Trio 3T MRI scanner at the Montreal Neurological Institute (MNI). Highresolution T1-weighted anatomical images with voxel size $=1 \mathrm{x} 1 \mathrm{x} 1$ $\mathrm{mm}$ were first obtained for anatomical localization. Then functional data were acquired with an echo-planar $\mathrm{T} 2 *$ weighted sequence for blood oxygenation level-dependent (BOLD) contrast (TR $=2.5 \mathrm{~s}$; $\mathrm{TE}=30 \mathrm{~ms} ;$ flip angle, $90^{\circ} ; \mathrm{FOV}=256 \mathrm{~mm}$, voxel size $=4 \times 4 \times 4 \mathrm{~mm}$, number of slices $=34$ ).
For each session, participants underwent three 11-min functional runs. During each run subjects passively viewed images of food or scenery, presented via a projector and a mirror placed on the head coil. We used a passive viewing task that we have utilized in previous stress (Dagher, Tannenbaum, Hayashi, Pruessner, \& McBride, 2009) and food reactivity studies (Malik, McGlone, Bedrossian, \& Dagher, 2008). The food and scenery images had been previously matched for visual appeal (Malik, McGlone, Bedrossian, \& Dagher, 2008). Each run was comprised of 72 unique images, 24 each of high and low-calorie foods (e.g. brownies, vegetables) and scenery (Fig. 1). The order of picture representation was randomized across subjects. Images were presented for $3.0 \mathrm{~s}$, for the first 11 subjects and $4.0 \mathrm{~s}$ for the last 11 subjects. The counterbalancing for order was balanced across the two phases so that the order of the scan was not confounded with the change in stimulus duration. Image presentation was followed by a fixation cross with a jittered interstimulus interval (2.5-6.0 s). Stimulus presentation was done using E-Prime (Psychology Software Tools Inc, Sharpsburg PA, USA).

\subsection{Imaging data analysis}

Each structural T1 image was first pre-processed with CIVET (version 2.0) (http://www.bic.mni.mcgill.ca/ServicesSoftware/ CIVET) (Ad-Dab'bagh et al., 2006) for non-uniformity correction (Sled, Zijdenbos, \& Evans, 1998) and linear transformation to Montreal Neurological Institute (MNI) space. A mask of the brain was generated using minc-BET (Smith, 2002).

Pre-processing of the BOLD data using FEAT (FMRI Expert Analysis Tool, Version 6.00, part of FMRIB's Software, www.fmrib. ox.ac.uk/fsl) consisted of slice timing and motion correction, spatial smoothing $(6 \mathrm{~mm})$, and high band-pass filtering with a cut- 
off frequency of $0.1 \mathrm{~Hz}$ (Mark Jenkinson, Beckmann, Behrens, Woolrich, \& Smith, 2012). Each functional volume was registered to the participant's high-resolution anatomical image linearly using FLIRT (Mark Jenkinson, Bannister, Brady, \& Smith, 2002; M Jenkinson \& Smith, 2001) and then to FSL's standard MNI template brain (MNI 152, T1 $2 \mathrm{~mm}$ ) nonlinearly using FNIRT (Andersson, Jenkinson, Smith, \& Andersson, 2007a, Andersson, Jenkinson, Smith, \& Andersson, 2007b).

The removal of artifacts due to head motion or pulse and respiration was performed prior to statistical analysis using FIX (FMRIB's ICA-based X-noiseifier) with the standard training data (Standard.Rdata) supplied with the software (Beckmann \& Smith, 2004Salimi-Khorshidi et al., 2014). Independently, two researchers classified the FIX components as noise or data based on their spatial appearance and frequency distribution in a subset of participants and confirmed that FIX only removed the noise components from the data.

A general linear model (GLM) was performed for the first-level statistical analysis of the BOLD time-series using FILM (Woolrich, Ripley, Brady, \& Smith, 2001). The regressors for the GLM were the high and low-calorie food and scenery image presentation and were modeled by convolving the time course with a double-gamma hemodynamic response function (HRF) and applying temporal filtering. The resulting contrast images (i.e. food minus scenery, high-calorie vs. low-calorie food images) were fed into a secondlevel fixed-effects analysis for each subject to average contrast estimates over runs within subjects by forcing random effects variance to zero in FLAME (FMRIB's Local Analysis of Mixed Effects) (Beckmann, Jenkinson, \& Smith, 2003; Woolrich, 2008; Woolrich, Behrens, Beckmann, Jenkinson, \& Smith, 2004). The t-stat maps for the contrast of interest at exam vs. non-exam condition were combined to across subjects for the third-level analysis.

In the third level analysis, we examined the results at the group level using non-parametric permutation tests $(n=5000$ permutations) with threshold-free cluster enhancement (TFCE) algorithm using randomise in FSL (Smith \& Nichols, 2009). In the final model, we controlled for sex and stimulus duration (3s vs. 4s) by including them as demeaned binary covariates in the model. We investigated how the BIS score and hunger levels affected the reactivity to food cues (i.e. food vs. scenery images or high-calorie vs. low-calorie food cues) by including them as regressors in the GLM model. Since we are interested in value related activity, we performed a region of interest analysis using a mask from a fMRI meta-analysis for subjective value at the time of decision (see Fig. 6A Bartra, McGuire, \& Kable, 2013). In addition, we investigated how the BIS score related to changes in BOLD in regions implicated in stress. For this purpose, we restricted our analysis to a regional forward inference mask associated with the term "stress" in the NeuroSynth database of fMRI studies (http:/neurosynth.org/analyses/terms/ stress/) (Yarkoni, Poldrack, Nichols, Van Essen, \& Wager, 2011).

\subsection{Functional connectivity}

Functional connectivity analysis was conducted using psychophysiological interaction (PPI) (O'Reilly, Woolrich, Behrens, Smith, \& Johansen-Berg, 2012). We used the mean BOLD time-series signal extracted from a $4 \mathrm{~mm}$ diameter seed region of interest (ROI) in the VmPFC. This seed was centered on the peak coordinate of the contrast of high-calorie vs. low-calorie contrast at the exam minus no exam condition that correlated with the BIS score (MNI: $\mathrm{x}=-2, \mathrm{y}=52, \mathrm{z}=-10$ ). The ROI was transformed from MNI space into each individual's functional space using a linear transformation (FLIRT). The mean time series of the seed ROI and the contrast of interest (i.e. high-calorie vs. low-calorie food cues) were multiplied to create the PPI regressor in the first level analysis.
Higher-level analysis was repeated as stated above. We restricted our analysis to a regional forward inference mask associated with the term "dlPFC" from NeuroSynth (http://neurosynth.org/ analyses/terms/dlpfc/) (Yarkoni et al., 2011).

For all of the univariate results reported here, the significance of the clusters was determined by threshold-free cluster enhancement (TFCE) with a significance level of $p<0.05$ family wise error rate (FWER) corrected after small volume correction (SVC) of the apriori hypothesized brain mask. All coordinates are given in MNI space.

\section{Results}

\subsection{Participants}

22 students participated in the study. All of the behavioural test scores, as well as BMI, were tested for normality using the Lilliefors test. The demographics of the study population are listed in Table 1. Subjects did not show significant change in BMI across the two sessions $(\mathrm{t}(21)=0.817, \mathrm{p}=0.423)$.

\subsection{Psychological measures}

Participants reported increased perceived stress during the academic exam period relative to the non-exam condition as measured by the PSS $(t(21)=2.3, p=0.03)$ (Fig. 2-A). In addition, VAS scales administered three times during the scanning session also demonstrated higher levels of perceived stress $(F(1,129)=18.7$, $\mathrm{p}<0.0001)$ and hunger $(\mathrm{F}(1,129)=3.82, \mathrm{p}=0.05)$ during the exam session. There was also a main effect of time as the scan progressed: participants reported reduced perceived stress $(F(1,129)=8.36$, $\mathrm{p}=0.005)$ and increased hunger $(\mathrm{F}(1,129)=8.33, \mathrm{p}=0.005)$ (Supplemental Fig. 1). Participants who felt on average more stressed as reported by the VAS scale also reported feeling on average hungrier $(\mathrm{r}=0.45, \mathrm{p}=0.038)$ and showed a reduced rate of drop in saliva cortisol levels assessed by AUCi $(r=-0.50, p=0.02)$ (Supplemental Fig. 2).

\subsection{Cortisol and ghrelin}

Saliva cortisol collected at home showed a significant main effect of time of day consistent with its circadian fluctuation $(\mathrm{F}(4,812)=102.04, \mathrm{p}<0.0001)$, but not of the exam-condition $(\mathrm{F}(1,812)=0.53, \mathrm{p}=0.47)$. There was a trend for an interaction between time of day and condition $(\mathrm{F}(4,812)=2.16, \mathrm{p}=0.072)$ (Fig. 2-C). CAR measurements, a marker for allostatic load, were not different across conditions (Fig. 2-D); for AUCg $\mathrm{F}(1,41)=0.15$, $\mathrm{p}=0.26$ and for AUCi $\mathrm{F}(1,41)=2.05, \mathrm{p}=0.16$ respectively. Salivary cortisol levels measured during the course of the scan did not show any significant effect of condition $\mathrm{F}(1,21)=0.76, \mathrm{p}=0.40$ or time $\mathrm{F}(4,18)=4.23, \mathrm{p}=0.103$ (Supplemental Fig. 1).

Table 1

Participant characteristics and psychological measures.

\begin{tabular}{lllll}
\hline $\mathrm{N}=22(13 \mathrm{~F}, 9 \mathrm{M})$ & Minimum & Maximum & Mean & Std. Deviation \\
\hline Age & 18 & 32 & 20.5 & 2.9 \\
TFEQ Restraint & 1 & 16 & 7.0 & 4.5 \\
TFEQ Disinhibition & 2 & 14 & 6.8 & 3.5 \\
TFEQ Hunger & 0 & 11 & 5.4 & 3.2 \\
BIS & 8 & 27 & 18.0 & 4.8 \\
BAS reward & 5 & 20 & 15.5 & 5.5 \\
BAS drive & 6 & 14 & 10.4 & 2.2 \\
BAS fun & 4 & 16 & 10.7 & 3.4 \\
BMI non-exam $\left(\mathrm{kg} / \mathrm{m}^{2}\right)$ & 19.5 & 26.0 & 22.6 & 1.9 \\
BMI exam $\left(\mathrm{kg} / \mathrm{m}^{2}\right)$ & 19.8 & 26.6 & 22.7 & 1.9 \\
\hline
\end{tabular}


A

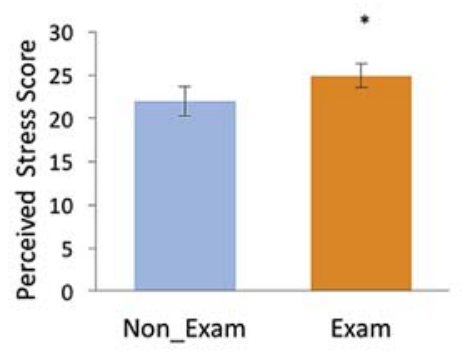

C

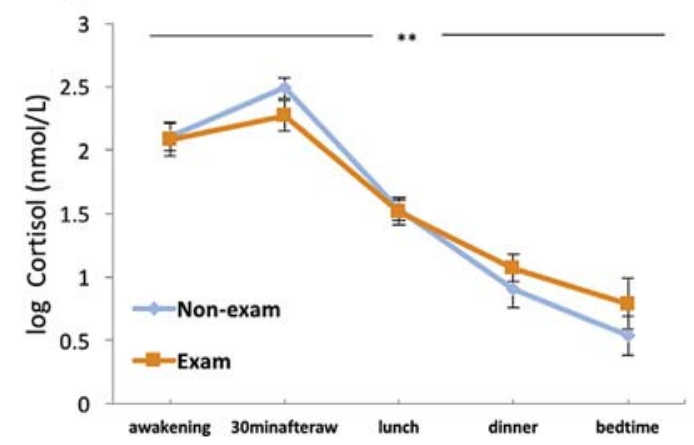

B

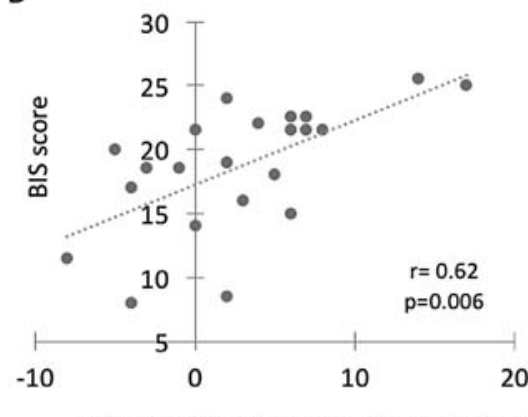

Change in PSS score (Exam minus NonExam)

D

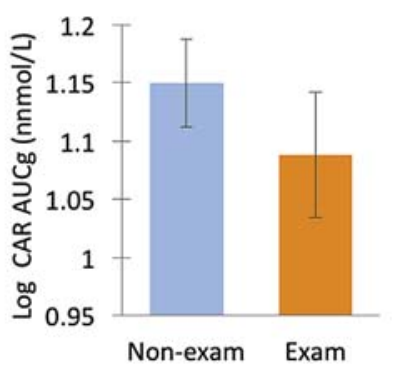

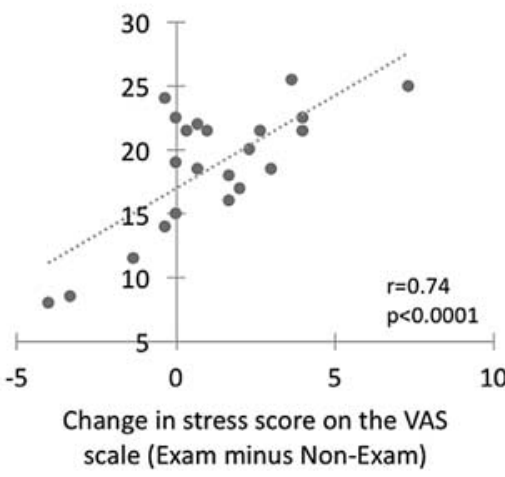

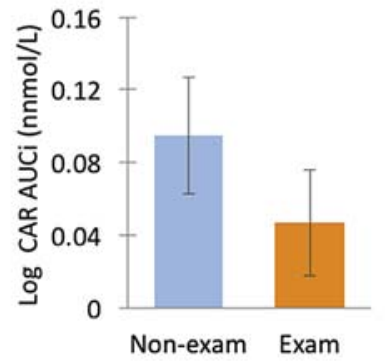

Fig. 2. Changes in Exam Condition Compared with the Non-Exam Condition.

(A) Perceived stress was significantly greater in the exam compared to the non-exam condition $(t(21)=2.3, p=0.03)$. (B) Individual changes in stress levels measured by PSS and VAS correlated with BIS score. (C) Diurnal salivary cortisol assessed on four days around each session. There was a significant main effect of time of day consistent with its circadian fluctuation $(F(4,812)=102.04, p<0.0001)$. There was no effect of condition. There was a trend for an interaction between time of day and condition $(F(4,812)=2.16$, $p=0.072$. $(D)$ Morning salivary cortisol with respect to ground (AUCg) and to baseline (AUCi). There was no effect of condition of AUCg and AUCi. Standard Errors of the Means displayed as error bars. CAR: cortisol awakening response. ${ }^{*} \mathrm{p}<0.05 ;{ }^{* *} \mathrm{p}<0.01$.

Plasma ghrelin levels showed a significant increase during the course of the scan (total ghrelin: $F(1,14)=19.9, \mathrm{p}=0.001$; acylghrelin: $F(1,14)=5.82, p=0.03)$. However, there was no significant effect of condition (total ghrelin: $F(1,14)=0.02, p=0.89$; acylghrelin: $\mathrm{F}(1,14)=0.281, \mathrm{p}=0.604)$ nor a time $\times$ condition interaction (total ghrelin: $\mathrm{F}(1,14)=0.082, \mathrm{p}=0.78$, acyl-ghrelin: $\mathrm{F}(1,14)=0.021, \mathrm{p}=0.89$ ) (Supplemental Fig. 1 ).

\subsection{Personality - stress interaction}

BIS score was correlated with the increase in stress levels during the exam condition compared to the non-exam condition measured using the PSS $(\mathrm{r}=0.62, \mathrm{p}=0.006)$ and the mean VAS stress score during the scan $(r=0.74, p<0.0001)$ (Fig. 2-B).

\section{5. fMRI results}

We first compared the presentation of food images (highcalorie and low-calorie) to scenery (control) images irrespective of the experimental condition. This resulted in increased BOLD signal in a set of brain structures implicated in appetite regulation and food intake (Supplementary Fig. 4; Supplemental Table 5): the visual cortex, the insula, the thalamus, the striatum, the amygdala, the OFC, the ACC and the inferior frontal gyrus (IFG). We then compared the response to high calorie versus low calorie pictures. There was increased BOLD in temporal and inferior fusiform gyrus, in occipital cortex and superior parietal lobule (not shown) irrespective of the condition

\section{(Supplemental Table 7).}

\section{6. fMRI results: correlation with BIS}

We analyzed whether, in the exam condition compared to the non-exam condition, participants' BIS scores related to changes in activity in regions associated with subjective value for the high calorie minus low calorie image contrast. For this purpose, we utilized a mask derived from a meta-analysis of subjective value (Bartra et al., 2013). In this ROI, participants' BIS scores correlated with increased activation in the vmPFC (Fig. 3-A; Supplemental Table 1). Controlling for the change in hunger between the conditions did not change this result (Supplemental Fig. 3-A). In addition, BIS scores also related to increased response to highcalorie vs. low-calorie food cues bilaterally in the amygdala in the exam minus non-exam condition using an ROI of stress-related regions derived from NeuroSynth (Fig. 3-C; Supplemental Table 1). We also repeated this analysis for food (high-calorie and lowcalorie) minus scenery contrast. BIS also related to increased reactivity in the vmPFC in exam minus non-exam condition for food minus scenery contrast (Supplementary Fig. 5; Supplemental Table 6).

\section{7. fMRI results: correlation with hunger}

Changes in hunger levels correlated with increased BOLD to high-calorie vs. low-calorie foods in the bed nucleus of the stria terminalis (BNST) in exam minus non-exam condition within the 
A

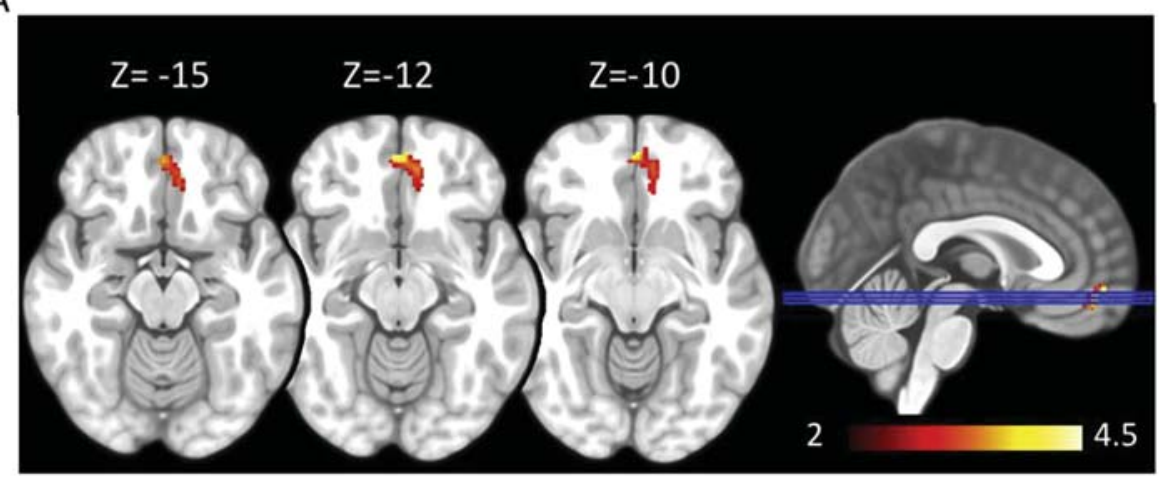

C

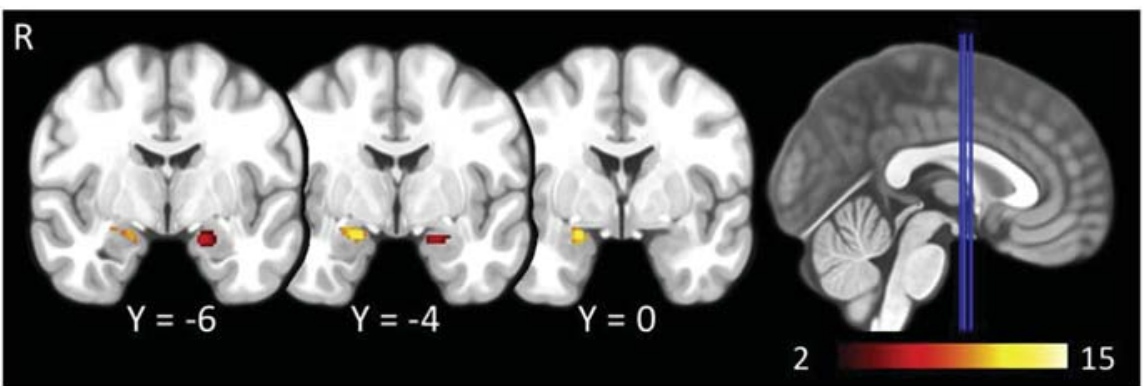

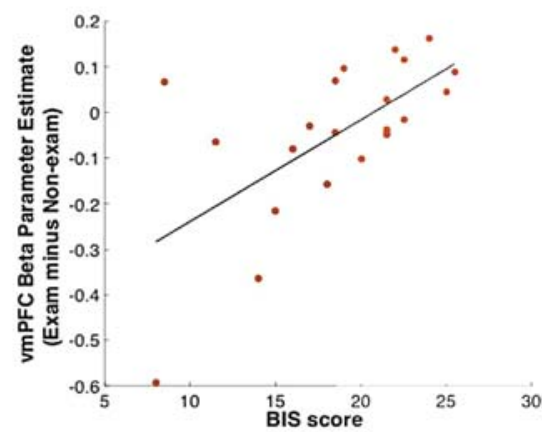

D

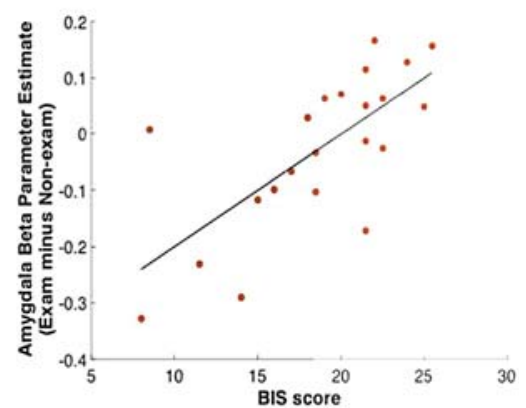

Fig. 3. BIS score relates to high-calorie food cue reactivity in the vmPFC and the amygdala in the exam compared with non-exam condition.

(A) Activation to high-calorie vs. low-calorie food cues at the final exams compared to the non-exam period correlated with the BIS score (p < 0.05 FWER, SVC). (B) Changes in the vmPFC region beta estimate to the high-calorie versus low-calorie food cues in the exam condition compared with the non-exam condition versus the BIS score. (C) BIS score was correlated with BOLD response in amygdala ( $p<0.05$ FWER, SVC). (D) Changes in amygdala beta estimate for the high-calorie vs low-calorie food contrast compared with participants' BIS score (Fig. 3-C; Supplemental Table 1). The beta estimates were derived from the entire cluster. The color scale represents t-statistics derived form 5000 permutations of the data. SVC: Small Volume Correction. FWER: Family-wise Error Rate. (For interpretation of the references to colour in this figure legend, the reader is referred to the web version of this article.)

\section{ROI mask of subjective value (Supplemental Fig. 3-B).}

\subsection{Changes in connectivity: correlation with BIS}

We next investigated the changes in functional connectivity between conditions with PPI using the $\operatorname{vmPFC}(\mathrm{MNI}: \mathrm{x}=-2, \mathrm{y}=52$, $\mathrm{z}=-10$ ) as a seed region based on the peak from the previous analysis. We chose this region because connectivity between vmPFC and dIPFC has been shown during self-regulation of foodchoice (Hare, Camerer, \& Rangel, 2009).

We did not observe an overall increase/decrease in connectivity in the exam vs. non-exam condition. However, when BIS score was used as a covariate, it was negatively correlated with the connectivity between the vmPFC and bilateral dIPFC (BA9/BA46) in the exam condition compared with the non-exam condition, for the high-calorie vs low-calorie food contrast (Fig. 4-A; Supplemental Table 3). Greater BIS score was associated with reduced functional connectivity at exam minus no exam, for the high calorie minus low calorie food contrast.

\section{Discussion}

We utilized the final exam period for university undergraduates to study the effects of psychosocial stress on brain mechanisms of appetite control. The exam period was associated with increased levels of perceived stress, and with individual differences at behavioural and neuronal levels. At the behavioural level, BIS predicted the increase in perceived stress as measured by the PSS and VAS, consistent with previous literature that links BIS to increased anxiety when faced with daily pressures (Everaerd, Klumpers, van
Wingen, Tendolkar, \& Fernández, 2015; Gudiño, 2013; van der Linden et al., 2007). This suggests that individuals with a high BIS score experience more stress during the final exam period (Fig. 5).

Stress has been documented to shift food preferences towards highly palatable foods (Dallman, 2010; Maier et al., 2015; Rudenga et al., 2012). In line with this, we found that BIS score related to increased BOLD during stress versus the non-stress period while viewing high-calorie food pictures compared to low-calorie pictures in the vmPFC and in the amygdala. These regions have been implicated in food reward. Specifically, the vmPFC is thought to encode the current value of food cues (Plassmann, O'Doherty, \& Rangel, 2007). In summary, during the stress period, brain areas that compute incentive salience demonstrated greater activation to high calorie food compared with low calorie food cues (Fig. 5).

This response to palatable food cues in the vmPFC may interact with reduced self-control to increase food consumption (Hare et al., 2009; Lawrence, Hinton, Parkinson, \& Lawrence, 2012). Stress has been linked to the reduced function of prefrontal networks that have been implicated in self-control (Arnsten, 2015). In the exam condition, participants who scored high on the BIS also showed reduced functional connectivity between the vmPFC and the bilateral dIPFC, when looking at high-calorie food images (Fig. 5). DIPFC has been shown to be functionally connected to value computing areas such as vmPFC and upregulated when exerting self-control during food choice (Hutcherson, Plassmann, Gross, \& Rangel, 2012). For example, increased activity in the dlPFC has been related to regulatory self-control and healthier food choice (Hollmann et al., 2012; Maier et al., 2015) via downregulation of the vmPFC signal (Hare et al., 2009). A similar effect has been recently shown using an acute physical (i.e. cold water) stressor, where 
A

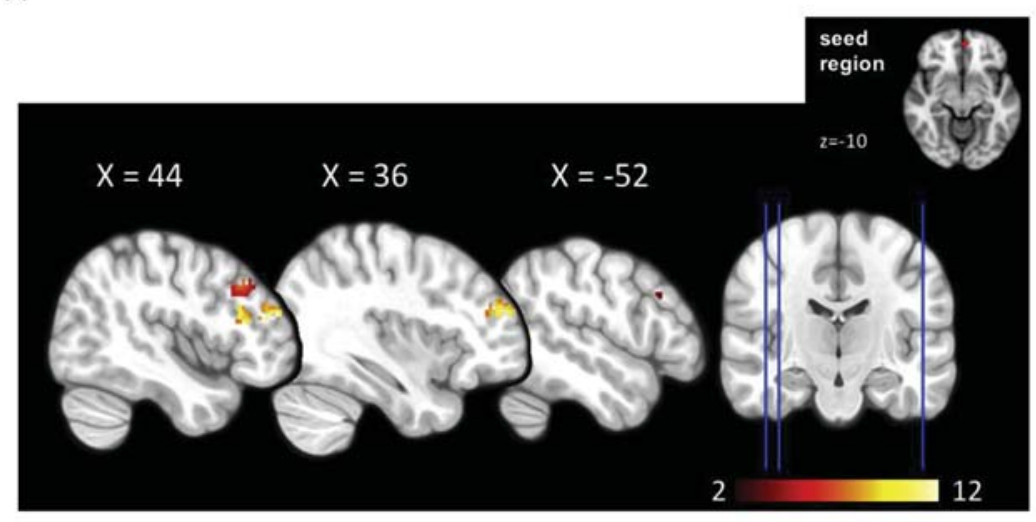

B

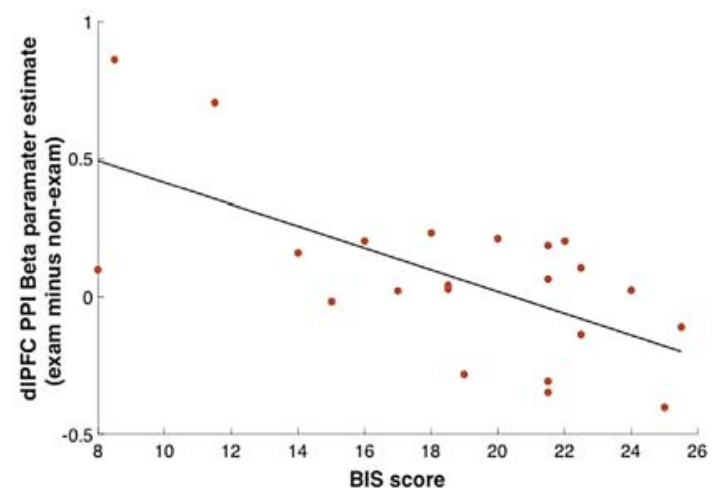

Fig. 4. BIS score relates to reduced connectivity from vmPFC to dIPFC in the exam vs. non-exam condition for the high-calorie versus low-calorie food contrast.

(A) PPI analysis with the vmPFC seed revealed that dIPFC connectivity is negatively related to the BIS score in the exam minus non-exam condition for the high-calorie vs. lowcalorie food contrast ( $<0.05$ FWER, SVC). (D) Changes in vmPFC connectivity with right dIPFC and left dIPFC cluster versus the BIS score. The beta estimates were derived from the entire cluster. The color scale represents t-statics derived from 5000 permutations of the data. SVC: Small Volume Correction. FWER: Family-wise Error Rate.

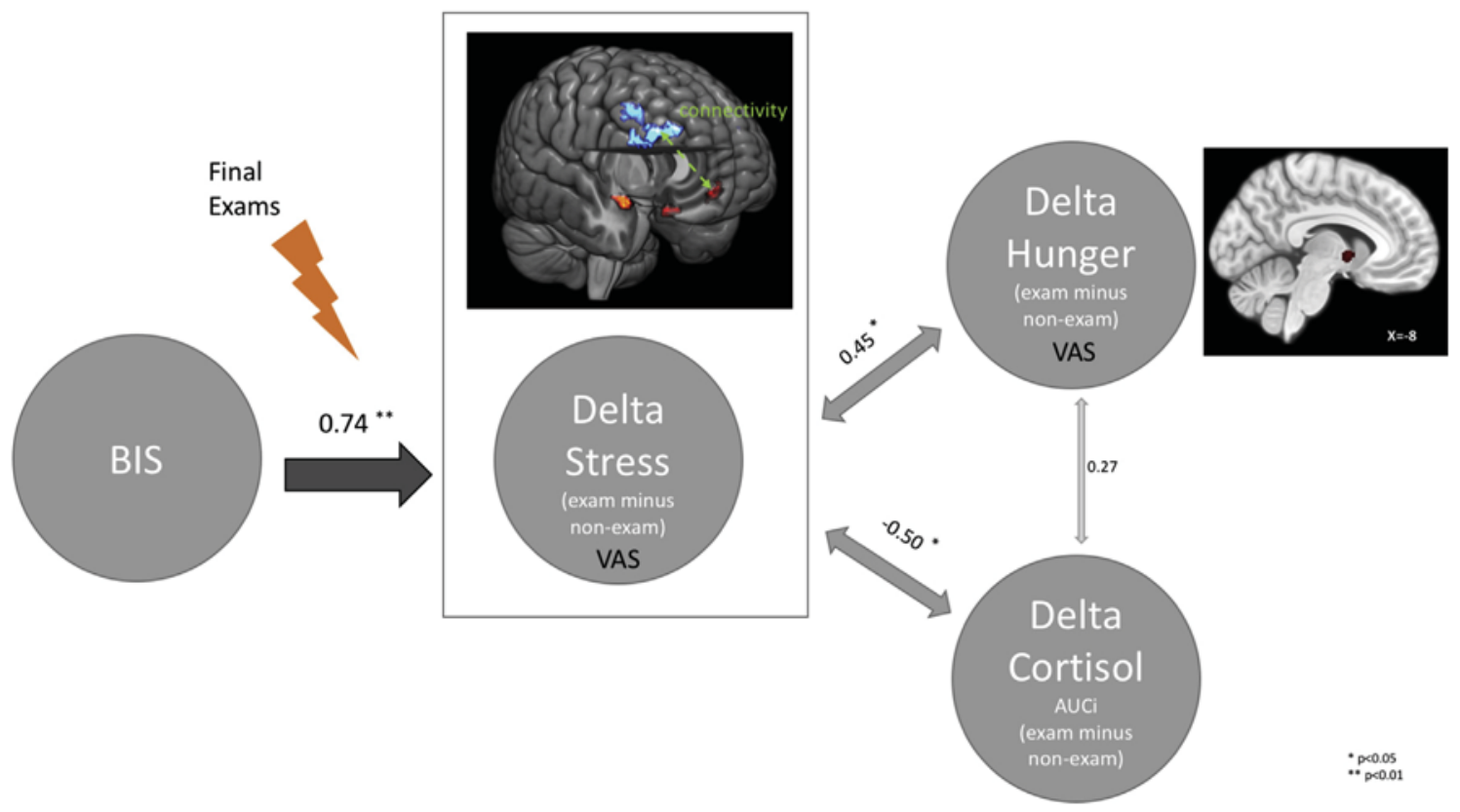

Fig. 5. Summary for Individual Differences in the Exam vs. Non-exam Condition.

Participants' BIS score correlated with the increases in the reported mean stress levels in the exam vs. non-exam condition measured using VAS ( $r=0.74$, $p<0.001$ ). In the brain, in the exam compared with the non-exam condition BIS scores related to increased BOLD signal in the vmPFC and in the amygdala to high-calories vs. low calorie food cues. vmPFC connectivity to dIPFC was negatively modulated by the BIS score. Increased stress during the final exams compared with non-exam condition also related to increased hunger levels measured using VAS. In the brain, hunger levels related to increased BOLD signal in the BNST to high-calories vs. low calorie food cues in the exam compared with the non-exam condition. Increased VAS stress also correlated with reductions in the cortisol levels in the exam compared with non-exam condition measured using AUCi. AUCi: Area under the curve with respect to baseline.

increases in perceived stress were related to reductions in the connectivity between the dIPFC and the vmPFC during food choice (Maier et al., 2015). Reduced prefrontal control of value signals and consequent increased food cue reactivity in stressed vulnerable individuals might result in increased impulsivity during food choice, favoring the selection of palatable calorically dense foods. This may underlie vulnerability to caloric overconsumption during stressful times.

Individuals who felt more stressed during the final exams also exhibited more hunger. The increase in the mean hunger levels in the exam minus non-exam condition related to increased activity to high calorie food cues in the BNST. BNST is key integrator of several motivational states, plays a crucial role in the stress response and has been implicated in stress-induced drug-relapse (Avery, Clauss, \& Blackford, 2016). Animal studies have shown that activation of BNST leads to overeating in well-fed mice(Jennings, Rizzi, Stamatakis, Ung, \& Stuber, 2013). Together these results suggest that BNST might play an important role in stress-induced eating. Our results are in line with this suggestion.

Activation of the HPA axis and subsequent changes in the endocrine system play an important role in mediating the stress response. In addition to cortisol, stress can also mediate secondary 
effects via the active form of ghrelin (acyl-ghrelin), a hunger hormone that increases the incentive value of food cues (Malik, McGlone, Bedrossian, \& Dagher, 2008). To address this, we analyzed how cortisol and ghrelin changed across conditions. In general, chronic stressors have been linked to a hypocortisolic profile (Tomiyama et al., 2011). Consistent with this, we observed a trend where diurnal cortisol levels were flatter (lower upon $30 \mathrm{~min}$ after awakening and higher at the bed-time) during the exam period (Fig. 2-B). Similarly, in laboratory cortisol measurements showed that increases in stress levels correlated negatively with the AUCi of cortisol in the exam compared with the non-exam condition. This suggests that increase stress levels might relate to flatter diurnal cortisol response. Cortisol is not only affected by stressors, but also by several factors such as sleep, time of day, alcohol and coffee consumption, steroid hormone intake and previous stress history (Kudielka, Hellhammer, Wüst, \& Kudielka, 2009). This could have contributed to the individual variability in cortisol measures and can explain why we failed to find a difference in cortisol levels during the exam period for the at-home measurements. In our study, we also did not see any significant differences across the conditions in ghrelin levels. This may have been result from our small sample size ( $\mathrm{n}=15$ for ghrelin), however it is consistent with previous reports (Rouach et al., 2007).

A strength of this study is that we studied currently non-obese undergraduate students preparing for final exams, perceived to be a highly stressful period yet limited in duration. This allowed us to utilize a within-subject design in a counter-balanced order to investigate how BIS can modulate stress reactivity. However, limitations of the study should be acknowledged. There might be a potential bias in our participant recruitment as students who experience very high levels of stress might have opted out from participating in this study. During the exam period students might have experienced changes in their sleep/eating schedules, which could have contributed to the observed effects. Although participants were asked to avoid caffeine on all test days, they may not have done so equally during the exam and non-exam periods. Our fMRI experiment did not include a food choice task. While we observed changes in food cue reactivity in regions associated with food choice, we did not investigate how stress reactivity and exam period interact to directly affect food choice, subsequent consumption, or weight gain. Because we employed a counterbalanced design (with half of the participants being tested initially at the exam condition), we did not have the power to test whether the BIS levels related to weight gain levels during the exam period.

\section{Conclusions}

Increased daily stress may lead vulnerable individuals to overeat. However, it has been particularly challenging to study stressinduced eating in humans. This is because there is a big variability in stress response and creating a stressor in the laboratory mimicking a real life situation is difficult. We overcame this challenge by utilizing a "real-life" experimental paradigm of a recurring and common stress in the lives of young adults that has well documented effects on eating behaviors and their consequences on body weight (Dallman, 2010; Groesz et al., 2012). Our results suggest that the variability in the stress response is related to the BIS personality trait. BIS scores are associated with stress vulnerability. This was observed as an increase in perceived stress levels and in neuronal measures of impulsivity towards high-calorie food cues similar to what is observed in obesity (Vainik et al., 2013). A personality-dependent impulsivity towards high-calorie food cues may explain why only some individuals overeat during stressful times and are susceptible to stress-induced obesity.

\section{Conflict of interest}

The authors declare no competing financial interests.

\section{Acknowledgments}

This work was supported by CIHR grants to AD and CIHR Frederick Banting and Charles Best Canada Graduate Scholarship to SN.

\section{References}

Ad-Dab'bagh, Y., Einarson, D., Lyttelton, O., Muehlboeck, J.-S., Mok, K., Ivanov, O., et al. (2006). The CIVET image-processing environment: A fully automated comprehensive pipeline for anatomical neuroimaging research. In M. Corbetta (Ed.), In proceedings of the 12th annual meeting of the organization for human brain mapping. Florence, Italy: Neurolmage.

Adam, T. C., \& Epel, E. S. (2007). Stress, eating and the reward system. Physiology \& Behavior, 91(4), 449-458. http://doi.org/10.1016/j.physbeh.2007.04.011.

Andersson, J. L. R., Jenkinson, M., Smith, S., \& Andersson, J. (2007a). Non-linear optimisation FMRIB Technial report TR07JA1.

Andersson, J. L. R. Jenkinson, M., Smith, S., \& Andersson, J. (2007b). Non-linear registration aka spatial normalisation FMRIB Technial report TR07JA2.

Arnsten, A. F. T. (2015). Stress weakens prefrontal networks: Molecular insults to higher cognition. Nature Neuroscience, 18(10), 1376-1385. http://doi.org/10. 1038/nn.4087.

Avery, S. N., Clauss, J. A., \& Blackford, J. U. (2016). The human BNST: Functional role in anxiety and addiction. Neuropsychopharmacology, 41(1), 126-141. http://doi. org/10.1038/npp.2015.185.

Bartra, O., McGuire, J. T., \& Kable, J. W. (2013). The valuation system: A coordinatebased meta-analysis of BOLD fMRI experiments examining neural correlates of subjective value. NeuroImage, 76, 412-427. http://doi.org/10.1016/j.neuroimage. 2013.02.063.

Beck A.T, B. R. W. (1972). Screening depressed patients in family practice: A rapid technique. Postgraduate Medicine, 52(6), 81-85.

Beckmann, C. F., Jenkinson, M., \& Smith, S. M. (2003). General multilevel linear modeling for group analysis in FMRI. Neurolmage, 20(2), 1052-1063. http://doi. org/10.1016/S1053-8119(03)00435-X.

Beckmann, C. F., \& Smith, S. M. (2004). Probabilistic independent component analysis for functional magnetic resonance imaging. IEEE Transactions on Medical Imaging, 23(2), 137-152. http://doi.org/10.1109/TMI.2003.822821.

Carver, C. S., \& White, T. L. (1994a). Behavioral inhibition, behavioral activation, and affective responses to impending reward and punishment: The BIS/BAS Scales. Journal of Personality and Social Psychology. http://doi.org/10.1037/0022-3514. 67.2.319.

Carver, C. S., \& White, T. L. (1994b). Behavioral inhibition, behavioral activation, and affective responses to impending reward and punishment: The BIS/BAS Scales. Journal of Personality and Social Psychology, 67(2), 319-333. http://doi.org/10. 1037/0022-3514.67.2.319.

Chang, E. C., Kahle, E. R., Yu, E. A., \& Hirsch, J. K. (2014). Behavioral inhibition system and behavioral activation system (BIS/BAS) motives and loneliness as predictors of eating disturbances in female college Students: Interpersonal context matters. Journal of Social and Clinical Psychology, 33(3), 250-269. http://doi.org/10. 1521/jscp.2014.33.3.250.

Cohen, S., Kamarck, T., \& Mermelstein, R. (1983). A global measure of perceived stress. Journal of Health and Social Behavior, 24, 385-396. http://doi.org/10. 2307/2136404.

Dagher, A. (2012). Functional brain imaging of appetite. Trends in Endocrinology and Metabolism: TEM, 23(5), 250-260. http://doi.org/10.1016/j.tem.2012.02.009.

Dagher, A., Tannenbaum, B., Hayashi, T., Pruessner, J. C., \& McBride, D. (2009). An acute psychosocial stress enhances the neural response to smoking cues. Brain Research, 1293, 40-48. http://doi.org/10.1016/j.brainres.2009.07.048.

Dallman, M. F. (2010). Stress-induced obesity and the emotional nervous system. Trends in Endocrinology and Metabolism, 21(3), 159-165. http://doi.org/10.1016/ j.tem.2009.10.004.

Epel, E., Lapidus, R., McEwen, B., \& Brownell, K. (2001). Stress may add bite to appetite in women: A laboratory study of stress-induced cortisol and eating behavior. Psychoneuroendocrinology, 26(1), 37-49. http://doi.org/10.1016/ S0306-4530(00)00035-4.

Everaerd, D. S., Klumpers, F., van Wingen, G., Tendolkar, I., \& Fernández, G. (2015). Association between neuroticism and amygdala responsivity emerges under stressful conditions. NeuroImage, 112, 218-224. http://doi.org/10.1016/j. neuroimage.2015.03.014.

Gray, J. A. (1987). The psychology of fear and stress. CUP Archive.

Groesz, L. M., McCoy, S., Carl, J., Saslow, L., Stewart, J., Adler, N., ... Epel, E. (2012). What is eating you? Stress and the drive to eat. Appetite, 58(2), 717-721. http:// 
doi.org/10.1016/j.appet.2011.11.028.

Gudiño, O. G. (2013). Behavioral inhibition and risk for posttraumatic stress symptoms in Latino children exposed to violence. Journal of Abnormal Child Psychology, 41(6), 983-992. http://doi.org/10.1007/s10802-013-9731-2.

Hare, T. A., Camerer, C. F., \& Rangel, A. (2009). Self-control in decision-making involves modulation of the vmPFC valuation system. Science (New York, N.Y.), 324(5927), 646-648. http://doi.org/10.1126/science.1168450.

Hollmann, M., Hellrung, L., Pleger, B., Schlögl, H., Kabisch, S. Stumvoll, M., ... Horstmann, A. (2012). Neural correlates of the volitional regulation of the desire for food. International Journal of Obesity (2005), 36(5) 648-655. http://doi.org/10.1038/ijo.2011.125.

Hutcherson, C. A., Plassmann, H., Gross, J. J., \& Rangel, A. (2012). Cognitive regulation during decision making shifts behavioral control between ventromedial and dorsolateral prefrontal value systems. The Journal of Neuroscience: The Official Journal of the Society for Neuroscience, 32(39), 13543-13554. http://doi org/10.1523/JNEUROSCI.6387-11.2012.

Jenkinson, M., Bannister, P., Brady, M., \& Smith, S. (2002). Improved optimization for the robust and accurate linear registration and motion correction of brain images. Neurolmage, 17(2), 825-841.

Jenkinson, M., Beckmann, C. F., Behrens, T. E. J., Woolrich, M. W., \& Smith, S. M. (2012). FSL. NeuroImage, 62(2), 782-790. http://doi.org/10.1016/j.neuroimage. 2011.09.015

Jenkinson, M., \& Smith, S. (2001). A global optimisation method for robust affine registration of brain images. Medical Image Analysis, 5(2), 143-156.

Jennings, J. H., Rizzi, G., Stamatakis, A. M., Ung, R. L., \& Stuber, G. D. (2013). The inhibitory circuit architecture of the lateral hypothalamus orchestrates feeding. Science (New York, N.Y.), 341(6153), 1517-1521. http://doi.org/10.1126/science. 1241812.

Keiser, H. N., \& Ross, S. R. (2011). Carver and whites' BIS/FFFS/BAS scales and domains and facets of the five factor model of personality. Personality and Individual Differences, 51, 39-44. http://doi.org/10.1016/j.paid.2011.03.007.

Kirschbaum, C., \& Hellhammer, D. H. (1994). Salivary cortisol in psychoneuroendocrine research: Recent developments and applications. Psychoneur oendocrinology, 19(4), 313-333.

Kudielka, B. M., Hellhammer, D. H., Wüst, S., \& Kudielka, B. M. (2009). Why do we respond so differently? Reviewing determinants of human salivary cortisol responses to challenge. Psychoneuroendocrinology, 34. http://doi.org/10.1016/j. psyneuen.2008.10.004.

Lawrence, N. S., Hinton, E. C., Parkinson, J. A., \& Lawrence, A. D. (2012). Nucleus accumbens response to food cues predicts subsequent snack consumption in women and increased body mass index in those with reduced self-control. NeuroImage, 63(1), 415-422. http://doi.org/10.1016/j.neuroimage.2012.06.070.

van der Linden, D., Taris, T. W., Beckers, D. G. J., \& Kindt, K. B. (2007). Reinforcemen sensitivity theory and occupational health: BAS and BIS on the job. Personality and Individual Differences, 42(6), 1127-1138. http://doi.org/10.1016/j.paid.2006. 07.029.

Maier, S. U., Makwana, A. B., \& Hare, T. A. (2015). Acute stress impairs self-control in goal-directed choice by altering multiple functional connections within the Brain's decision circuits. Neuron, 87(3), 621-631. http://doi.org/10.1016/j. neuron.2015.07.005.

Malik, S., McGlone, F., Bedrossian, D., \& Dagher, A. (2008a). Ghrelin modulates brain activity in areas that control appetitive behavior. Cell Metabolism, 7(5), 400-409. http://doi.org/10.1016/j.cmet.2008.03.007.

Malik, S., McGlone, F., Bedrossian, D., \& Dagher, A. (2008b). Ghrelin modulates brain activity in areas that control appetitive behavior. Cell Metabolism, 7(5) 400-409. http://doi.org/10.1016/j.cmet.2008.03.007.

McEwen, B. S., Bowles, N. P., Gray, J. D., Hill, M. N., Hunter, R. G., Karatsoreos, I. N. et al. (2015). Mechanisms of stress in the brain. Nature Neuroscience, 18(10) 1353-1363. http://doi.org/10.1038/nn.4086.

Newman, E., O'Connor, D. B., \& Conner, M. (2007). Daily hassles and eating behaviour: The role of cortisol reactivity status. Psychoneuroendocrinology, 32(2), 125-132. http://doi.org/10.1016/j.psyneuen.2006.11.006.

O'Reilly, J. X., Woolrich, M. W., Behrens, T. E. J., Smith, S. M., \& Johansen-Berg, H. (2012). Tools of the trade: Psychophysiological interactions and functional connectivity. Social Cognitive and Affective Neuroscience, 7(5), 604-609. http:// doi.org/10.1093/scan/nss055.

Pemberton, C., Wimalasena, P., Yandle, T., Soule, S., \& Richards, M. (2003). C- terminal pro-ghrelin peptides are present in the human circulation. Biochemical and Biophysical Research Communications, 310, 567-573. http://doi.org/10.1016/ j.bbrc.2003.09.045.

Plassmann, H., O'Doherty, J., \& Rangel, A. (2007). Orbitofrontal cortex encodes willingness to pay in everyday economic transactions. The Journal of Neuroscience: The Official Journal of the Society for Neuroscience, 27, 9984-9988. http:// doi.org/10.1523/JNEUROSCI.2131-07.2007.

Pruessner, J. C., Kirschbaum, C., Meinlschmid, G., \& Hellhammer, D. H. (2003). Two formulas for computation of the area under the curve represent measures of total hormone concentration versus time-dependent change. Psychoneuroendocrinology, 28(7), 916-931.

Pruessner, J. C., Wolf, O. T., Hellhammer, D. H., Buske-Kirschbaum, A., Von Auer, K. Jobst, S., ... Kirschbaum, C. (1997). Free cortisol levels after awakening: A reliable biological marker for the assessment of adrenocortical activity. Life Sciences, 61, 2539-2549. http://doi.org/10.1016/S0024-3205(97)01008-4.

Rouach, V., Bloch, M., Rosenberg, N., Gilad, S., Limor, R., Stern, N., et al. (2007). The acute ghrelin response to a psychological stress challenge does not predict the post-stress urge to eat. Psychoneuroendocrinology, 32(6), 693-702. http://doi. org/10.1016/j.psyneuen.2007.04.010.

Rudenga, K. J., Sinha, R., \& Small, D. M. (2012). Acute stress potentiates brain response to milkshake as a function of body weight and chronic stress. International Journal of Obesity, 37(2), 309-316. http://doi.org/10.1038/ijo 2012.39.

Salimi-Khorshidi, G., Douaud, G., Beckmann, C. F., Glasser, M. F., Griffanti, L., \& Smith, S. M. (2014). Automatic denoising of functional MRI data: Combining independent component analysis and hierarchical fusion of classifiers. NeuroImage, 90, 449-468. http://doi.org/10.1016/j.neuroimage.2013.11.046.

Sled, J. G., Zijdenbos, A. P., \& Evans, A. C. (1998). A nonparametric method for automatic correction of intensity nonuniformity in MRI data. IEEE Transactions on Medical Imaging, 17(1), 87-97. http://doi.org/10.1109/42.668698.

Smith, S. M. (2002). Fast robust automated brain extraction. Human Brain Mapping, 17(3), 143-155. http://doi.org/10.1002/hbm.10062.

Smith, S. M., \& Nichols, T. E. (2009). Threshold-free cluster enhancement: Addressing problems of smoothing, threshold dependence and localisation in cluster inference. Neurolmage, 44(1), 83-98. http://doi.org/10.1016/j. neuroimage.2008.03.061.

Stunkard, A. J., \& Messick, S. (1985). The three-factor eating questionnaire to measure dietary restraint, disinhibition and hunger. Journal of Psychosomatic Research, 29, 71-83. http://doi.org/10.1016/0022-3999(85)90010-8.

Tomiyama, A. J., Dallman, M. F., \& Epel, E. S. (2011). Comfort food is comforting to those most stressed: Evidence of the chronic stress response network in high stress women. Psychoneuroendocrinology, 36, 1513-1519. http://doi.org/10.1016 j.psyneuen.2011.04.005.

Tryon, M. S., Carter, C. S., Decant, R., \& Laugero, K. D. (2013). Chronic stress exposure may affect the brain's response to high calorie food cues and predispose to obesogenic eating habits. Physiology \& Behavior, 120, 233-242. http://doi.org/ 10.1016/j.physbeh.2013.08.010.

Vainik, U., Dagher, A., Dubé, L., \& Fellows, L. K. (2013). Neurobehavioural correlates of body mass index and eating behaviours in adults: A systematic review. Neuroscience and Biobehavioral Reviews. http://doi.org/10.1016/j.neubiorev.2012. 11.008 .

Verdejo-Garcia, A. (2014). Reward, reinforcement, and impulsivity in obesity. Treatment of the Obese Patient.

Wardle, J., Chida, Y., Gibson, E. L., Whitaker, K. L., \& Steptoe, A. (2011). Stress and adiposity: A meta-analysis of longitudinal studies. Obesity (Silver Spring, Md.), 19(4), 771-778. http://doi.org/10.1038/oby.2010.241.

Woolrich. (2008). Robust group analysis using outlier inference. NeuroImage, 41(2), 286-301. http://doi.org/10.1016/j.neuroimage.2008.02.042.

Woolrich, M. W., Behrens, T. E. J., Beckmann, C. F., Jenkinson, M., \& Smith, S. M (2004). Multilevel linear modelling for FMRI group analysis using Bayesian inference. NeuroImage, 21(4), 1732-1747. http://doi.org/10.1016/j.neuroimage. 2003.12.023.

Woolrich, M. W., Ripley, B. D., Brady, M., \& Smith, S. M. (2001). Temporal autocorrelation in univariate linear modeling of FMRI data. Neurolmage, 14(6) 1370-1386. http://doi.org/10.1006/nimg.2001.0931.

Yarkoni, T., Poldrack, R. A., Nichols, T. E., Van Essen, D. C., \& Wager, T. D. (2011). Large-scale automated synthesis of human functional neuroimaging data. $\mathrm{Na}$ ture Methods, 8(8), 665-670. http://doi.org/10.1038/nmeth.1635. 